\title{
Association Between PDLI Genetic Variation and Efficacy of Apatinib Monotherapy in Patients with Previously Treated Advanced NSCLC: A Real-World Retrospective Study
}

\section{Wenxia $\mathrm{Hu}$ \\ Bin Li \\ Nan Geng (D) \\ Xin $\mathrm{He}$ \\ Hui Ge \\ Ping Wang \\ Cuimin Ding}

Department of Respiratory Medicine, Fourth Hospital of Hebei Medical University, Shijiazhuang 0500I I, People's Republic of China
Correspondence: Cuimin Ding Department of Respiratory Medicine, Fourth Hospital of Hebei Medical

University, 12 Jian-Kang Road,

Shijiazhuang, 050000, People's Republic of China,

Tel +86 139-3385-2787

Email 13933852787@I63.com
Background: This study aimed to explore associations between $P D L 1$ polymorphisms and efficacy of apatinib for patients with previously treated advanced non-small cell lung cancer (NSCLC) in a real-world setting.

Methods: We retrospectively recruited 148 patients with previously treated advanced NSCLC from January 2015 to December 2019 continuously. Clinical efficacy in patients receiving apatinib treatment was evaluated. Adverse reactions were documented during treatment. Biological specimens of peripheral blood and cancer tissue biopsies were obtained for the genotyping of genetic variations in $P D L 1$ and corresponding gene-mRNA expression, respectively. Univariate association analysis between the status of PDL1 genetic variations and survival was performed with Kaplan-Meier survival analysis.

Results: The objective response rate of the 148 patients was $17.6 \%$ and disease-control rate $68.9 \%$. Prognostic data suggested that median progression-free survival (PFS) was 3.8 (95\% CI 3.13-4.47) months and median overall survival (OS) 10.5 (95\% CI 9.06-11.95) months. Regarding PDL1 genetic variation, only rs2297136 was of clinical significance. Prognosis analysis revealed that PFS and OS for the rs2297136 genotype were significantly different. Median PFS of patients with TC/CC and TT genotypes was 3 and 4.5 months, respectively $(P=0.006)$. Median OS of the two genotypes was 9 and 11.6 months, respectively $(P=0.04)$. Furthermore, the safety profile suggested that the most common adverse reactions were hypertension, dermal toxicity, fatigue, and oral toxicity. This study failed to find any significant association between adverse reactions and rs2297136. Interestingly, mRNAexpression analysis demonstrated that mRNA expression of PDL1 in biopsy cancer-tissue specimens was significantly different based on rs2297136-genotype status $(P<0.001)$.

Conclusion: The PDL1 polymorphism rs2297136 could be used as a potential biomarker for the prognosis of patients with NSCLC receiving apatinib monotherapy.

Keywords: NSCLC, apatinib, $P D L 1$, genetic variation, clinical outcome, safety, biomarker

\section{Background}

Lung cancer is the most common solid tumor and has the highest morbidity and mortality worldwide. According to the latest cancer-epidemiology data, 0.815 million new cases and 0.715 million new deaths due to lung cancer are observed in China currently. ${ }^{1}$ As the most common histological subtype, non-small cell lung cancer (NSCLC) accounts for approximately $85 \%$ in lung cancer. ${ }^{2}$ 
Recently, targeted drugs with different mechanisms of action were developed based on common somatic genemutation profiles with significant survival benefits. ${ }^{3}$ Unfortunately, approximately half the patients with NSCLC in China lack one potentially significant drivergene mutation, and correspondingly only platinum-based chemotherapy regimens are available as first-line treatment. ${ }^{4}$ Chemotherapy efficacy is dismal, with objective response rate (ORR) $20 \%-30 \%$ and median progression-free survival (PFS) 4-5 months. ${ }^{5}$ Single-agent chemotherapy with docetaxel, pemetrexed, gemcitabine, and immunotherapy are alternative second-line treatment options, with additional benefits in prolonging survival after first-line treatment. ${ }^{6}$

However, the advantages of traditional second-line treatment are limited currently. Immunotherapy drugs have been licensed in recent years, and the low ORR of $P$ D1/PDL1 inhibitors has exhibited that patients who could benefit from an immunotherapy drug are thus also limited. ${ }^{7}$ Consequently, patients with advanced NSCLC are in urgent need of therapeutic drugs upon disease progression after standard treatment. Angiogenesis was identified as an important therapeutic strategy for solid tumors and was proven to be an essential condition in the process of tumor growth according to the theory of Folkman in 1971. ${ }^{8}$ Antiangiogenic drugs exhibit potential anticancer activity in treatment show advanced NSCLC. Bevacizumab and ramucirumab exhibit potential survival benefits for patients with NSCLC as first-line and secondline treatment, respectively. ${ }^{9}$ In terms of antiangiogenesis multitargeted tyrosine-kinase inhibitors (TKIs), anlotinib has been a standard regimen for third-line treatment in China since 2018. ${ }^{10}$ As a similar antiangiogenesis TKI, apatinib has been approved as subsequent-line treatment for advanced or metastatic gastric cancer. ${ }^{11}$ Considerable research has been done on apatinib in the treatment of NSCLC, colorectal cancer, and breast cancer. ${ }^{12}$ Interestingly, as far as we know, the ORR of antiangiogenic targeted drugs was disappointing. The ORR of sorafenib, anlotinib, and fruquintinib monotherapy as thirdline treatment of advanced NSCLC is only $4.9 \%, 9.18 \%$, and $16.4 \%$, respectively. ${ }^{13}$ Consequently, great individual differences regarding the efficacy of antiangiogenic targeted drugs have been observed clinically. Therefore, exploration of biomarkers that could predict the efficacy of apatinib is necessary.

$P D L 1$ is one of the most important biomarkers for the prognostic prediction of immunotherapy. ${ }^{14}$ A study has shown that higher $P D L 1$ expression predicts superior clinical outcome with $P$ D $1 / P D L 1$ inhibitors. ${ }^{15}$ Although patients with positive $P D L 1$ expression can benefit from $P \mathrm{D} 1 / P \mathrm{DL} 1$ inhibitors, the association between the prognosis of patientsadministered antiangiogenic targeted drugs and $P D L 1$ expression status remains unknown. ${ }^{16}$ Wu et al investigated the association between PDL1 polymorphisms and prognoses of patients with gastric cancer. The CC genotype of rs 822336 resulted in superior prognoeis for all GC patients and those without postoperative chemotherapy. ${ }^{17}$ Interestingly, a recent study $\mathrm{Su}$ et al investigated the association between $P D L 1$ genetic variation and the prognosis of patients with R0-resection colorectal cancer who received capecitabine-based adjuvant chemotherapy in the real world. The conclusion indicated that the PDL1 $901 \mathrm{~T}>\mathrm{C}$ polymorphism might be a valuable biomarker for patients with CRC receiving capecitabinebased adjuvant chemotherapy. ${ }^{18}$ Furthermore, a prior study indicated that VEGFR inhibitors could possess immunomodulatory properties that might result in antitumor activity and reduce the function of regulatory $\mathrm{T}$ cells and CD14-positive immunosuppressive monocytes, thus increasing cytokine production from effector $\mathrm{T}$ cells in response to antigen stimulation and activating the innate immunoresponse. ${ }^{19}$ Consequently, VEGFR inhibitors could potentially play an synergetic role with immunotherapy.

However, the association between PDL1 genetic variation and efficacy in patients with advanced NSCLC who have received apatinib treatment is unknown. Consequently, our study aimed to investigate the association between $P D L 1$ genetic variation and the efficacy of apatinib monotherapy in patients with previously treated advanced NSCLC. Additionally, the potential correlation between $P D L 1$ genetic variations and $P D L 1$ gene-mRNA expression in biopsied cancer-tissue specimens was explored.

\section{Methods}

\section{Design}

Given that apatinib has been approved in Chinese mainland for 5 years, with considerable numbers of patients receiving apatinib treatment, our study was designed as a retrospective analysis. Consequently, patients with advanced NSCLC who had failed after previous systemic treatment from January 2015 to December 2019 in the Department of Respiratory Medicine of the Fourth 
Hospital of Hebei Medical University participated. Eligibility criteria were diagnosis of NSCLC with pathological staging of IIIB or IV, age $\geq 18$ years Eastern Cooperative Oncology Group (ECOG) performance status $0-2$, apatinib monotherapy used for those who had failed after or were unable to tolerate previous systemic standard treatment, at least one measurable target lesion based on response evaluation criteria in solid tumors (RECIST 1.1) available, and appropriate renal, hepatic, and hematopoietic function to receive apatinib treatment. Exclusion criteria were new diagnosis or uncontrollable central nervous system metastases, concomitant with other tumors or serious diseases, hemoptysis $>50 \mathrm{~mL} /$ day, and efficacy data evaluation not available. The flow chart of this study is given in Figure 1. Finally, a total of 148 patients with advanced NSCLC was enrolled in this study. The primary focus of this study was associations between $P D L 1$ polymorphisms, objective response rate (ORR), progression free survival (PFS), overall survival (OS), and adverse reactions. The study was conducted in accordance with the Declaration of Helsinki and approved by the ethics committee of the Fourth Hospital of Hebei Medical University. Informed consent was obtained from each enrolled patient.

\section{Administration of Apatinib}

Initial dosage of apatinib was $500 \mathrm{mg}$ or $750 \mathrm{mg}$ per day orally continuously for 28 days until disease progression or intolerable adverse reactions. Dosage was determined based on baseline physical conditions: body-surface area (BSA), ECOG score, and age, which was up to the clinical experience of the investigators. Dosage was adjusted based on the

From January 2015 to December 2019, a total of 316 patients with advanced NSCLC who failed after the standard treatment were underwent retrospective eligibility screening

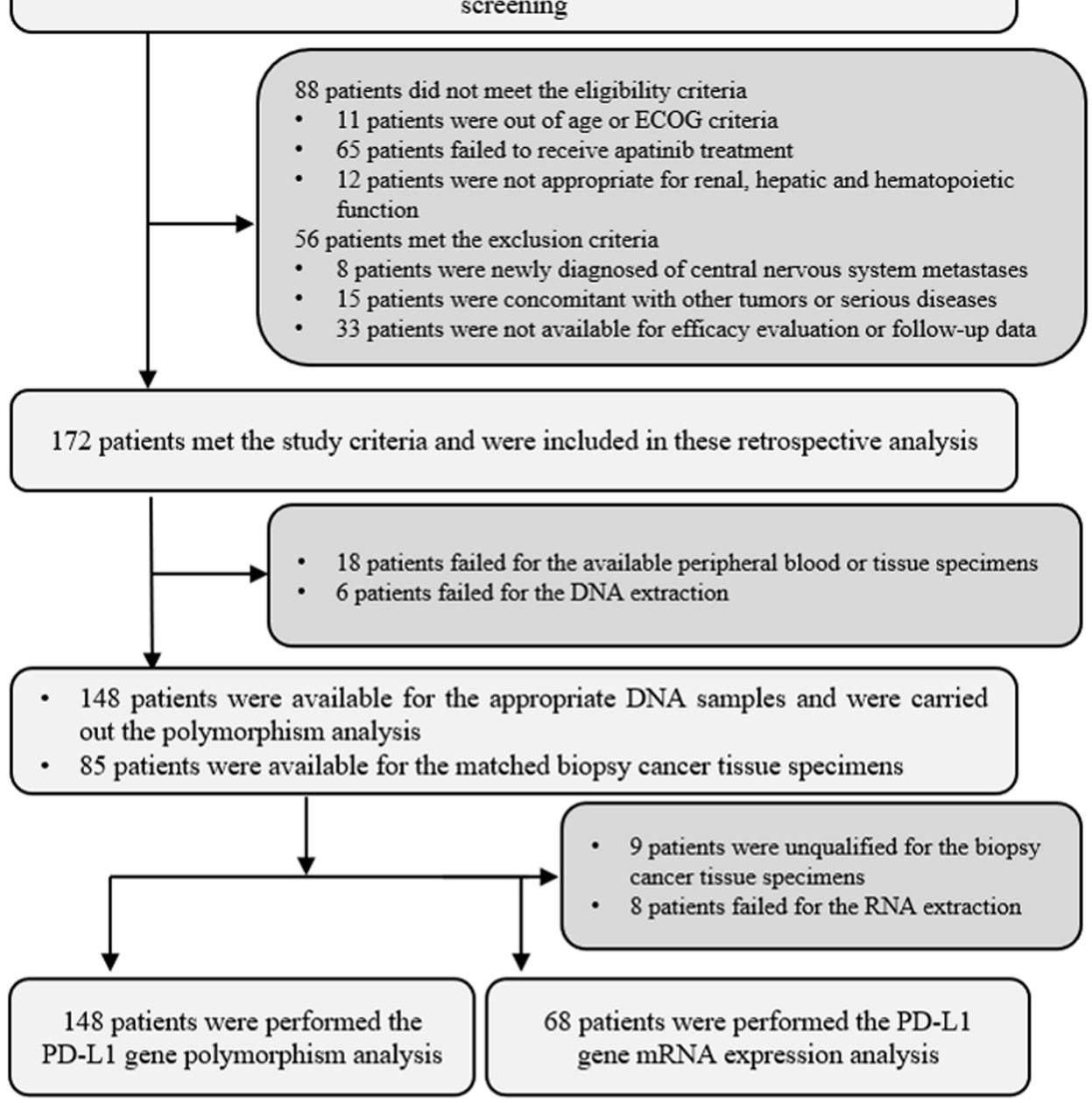

Figure I Flowchart of the study. 
hematologic or nonhematologic toxicity during treatment. Treatment was suspended when life-threatening toxic reactions occurred. Efficacy was assessed based on RECIST 1.1 criteria. ${ }^{20}$ Changes in target lesions were assessed after completion of the first cycle with computed-tomography scans and then every two cycles or when necessary. Safety profiles during treatment were documented using Common Terminology Criteria for Adverse Events version 4.03 to record hematologic and nonhematologic events that might be drug-related. ${ }^{21}$ Adverse reactions with incidence of $\geq 10 \%$ were recorded and analyzed.

\section{Collection of Peripheral Blood Specimens and Genotyping of PDLI Genetic Variation}

Approximately $4 \mathrm{~mL}$ peripheral blood prior to apatinib treatment from each patient was collected and genomic DNA extracted using phenol chloroform. In terms of the PDL1-polymorphism analysis, those included were rs2297136, rs822336, and rs822337. However, only rs2297136 was significantly associated with clinical outcomes on preliminary analysis. Consequently, following analysis was performed based on rs2297136-genotype status. The rs2297136 polymorphism was genotyped using PCRRFLP. The PCR product of rs2297136 was amplified firstly, with forward primer 5'-GCTCCCTGTTTGACTCCATC-3' and reverse primer 5'- TTTTTCCCCAGACCACTTCC-3'. The product was $274 \mathrm{bp}$, and a $2 \mu \mathrm{L}$ PCR products were digested using the restriction enzyme ApaI (Thermo Fisher Scientific, Waltham, MA, USA). Polymorphism genotypes were determined by the size of PCR bands: TT genotype, one 274 bp band; TC genotype, one 274 bp band, one 158 bp band, and one 116 bp band; and CC genotype, one 158 bp band and one 116bp band. Some genotyping results for rs2297136 were confirmed using ionizationtime-of-flight mass spectrometry (Sequenom, SanDiego, CA, USA).

\section{Collection of Cancer-Tissue Specimens and Analysis of PDLI-mRNA Expression}

Cancer tissue-specimens were collected from 85 randomly selected samples from the 148 patients. A total of 68 specimens were available for subsequent analysis and preserved in liquid nitrogen. All RNA samples were extracted using Trizol reagents and stored at $-80^{\circ} \mathrm{C}$ for $\mathrm{mRNA}$-expression analysis. A total of $500 \mathrm{ng}$ RNA extracted from the specimens was used as the template for reverse-transcription PCR to prepare the first stand of cDNA using a PrimeScript RT reagent kit.
Relative quantitative analysis of PDL1-mRNA expression was carried out using a LightCycler 480 withn SYBR premix Ex Taq system. The forward primer was 5'TTCAATGTGACCAGCACACTGAG-3', the reverse primer 5'-TTTTCACATCCATCATTCTCCCT-3'. PDL1-mRNA expression was detected using comparative $2^{-\triangle \triangle \mathrm{Ct}}$. GAPDHmRNA expression was used as an endogenous control.

\section{Statistical Analysis}

Variables in this study were analyzed using SPSS 25.0. Significance of proportional and continuous variables based on rs2297136-genotype status was assessed using the $\# x 1 D 712 ;^{2}$ and Mann-Whitney $U$ nonparametric tests, respectively. Primary analysis was performed on correlations between PFS and rs2297136-genotype status. Kaplan-Meier curves were used to draw the PFS and OS curves based on rs2297136-genotype status. Survival differences were compared using log-rank tests. PFS was defined as time from apatinib administration to progression or death from any cause, whichever occurred first. OS was defined as time from apatinib administration to death from any cause. For those without progression or death by the end of the study follow-up, survival end points were censored as per the date of last follow-up. ${ }^{22}$ Cox regression was performed for PFS in the multivariate analysis. $P<0.05$ was considered statistically significant.

\section{Results}

\section{Baseline Characteristics and Genotypes of PDLI-rs2297 I 36 Polymorphism}

Baseline characteristics of the 148 patients are shown in Table 1. Median age was 58 (28-79) years, 96 patients were men (64.86\%). Pathological stage IV was observed in 140 patients $(94.59 \%)$. ECOG 0 score was noted in 39 patients $(26.35 \%)$. Nonsmokers/former smokers numbered $107(72.30 \%)$. The most common histology of the patients was adenocarcinoma, with 114 cases $(77.03 \%)$, and squamous-cell carcinoma, with 28 cases $(18.92 \%)$. Numbers of patients with fewer than or equal to three and greater than three sites were 92 and 56, respectively. Positive drivergene mutation status was confirmed in 56 patients (37.84\%): 50 patients had EGFR mutations, four $A L K$ rearrangements, and two ROS1 rearrangements. History of treatment with second and further lines was observed in 43 cases and 105 cases, respectively. A total of 92 patients $(62.16 \%)$ had received targeted drug therapy. Interestingly, ten patients $(6.76 \%)$ had received 
Table I Baseline characteristics based on PDLI rs2297I36 genotype status

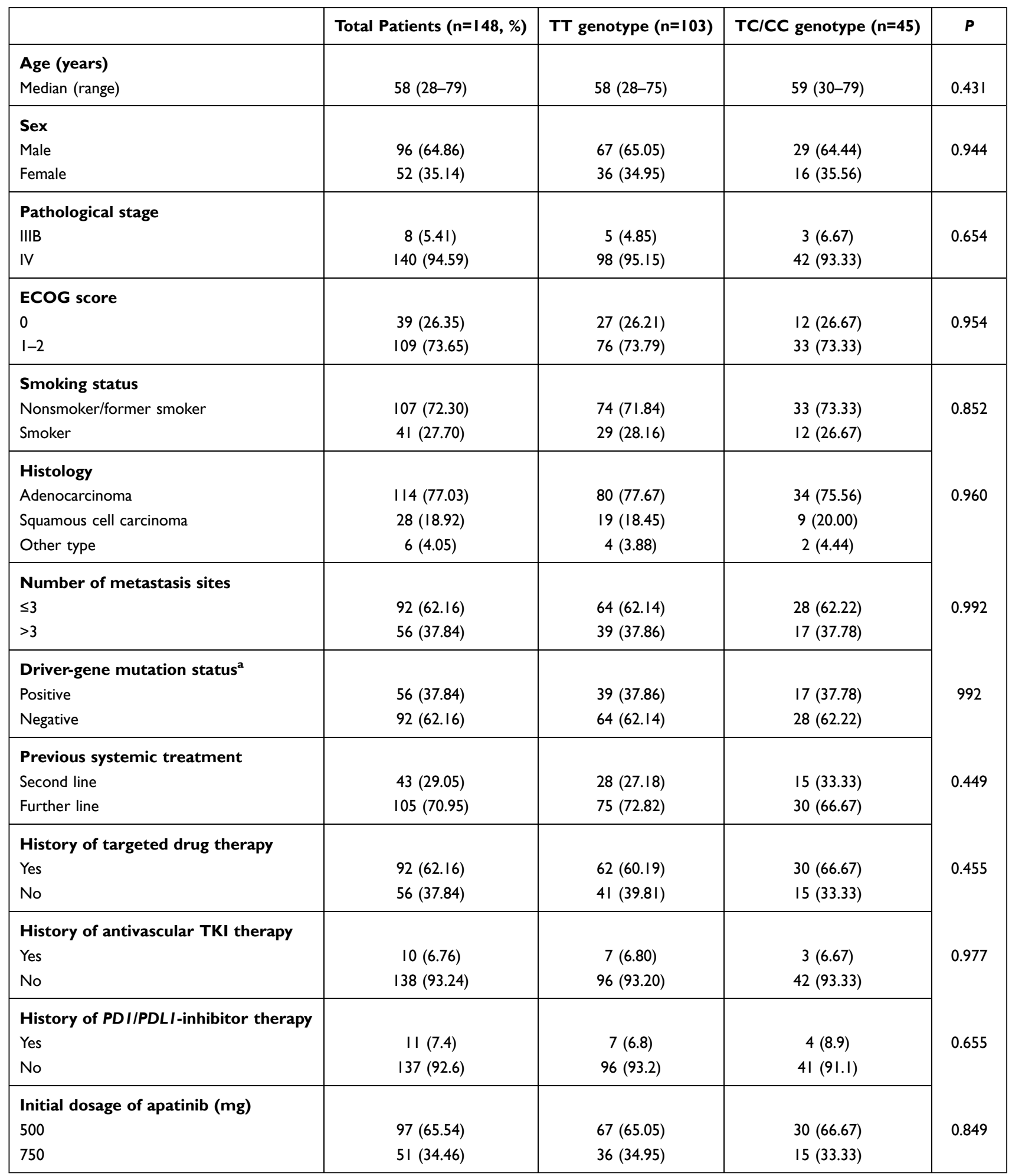

Note: ${ }^{a} E G F R$ mutation or ALK or ROSI rearrangements.

Abbreviations: NSCLC, non-small cell lung cancer; ECOG, Eastern Cooperative Oncology Group; TKI, tyrosine-kinase inhibitor.

antivascular TKI treatment. It should be noted that eleven patients had been treated with $P \mathrm{D} 1 / P \mathrm{DL} 1$ inhibitors. Initial dosage of $500 \mathrm{mg}$ and $750 \mathrm{mg}$ of apatinib was observed in 97 and 51 patients, respectively. rs2297136 was of clinical significance on subsequent analysis. The germ-line mutation frequency of rs 2297136 were TT genotype 103 cases (69.59\%), TC genotype 40 (27.03\%), and CC genotype five (3.38\%), with minor-allele frequency 0.17 . 
Distribution of the three genotypes was in accordance with the Hardy-Weinberg Equilibrium $(P=0.649)$. In view of the relatively rare frequency of the $\mathrm{CC}$ genotype, $\mathrm{CC}$ and $\mathrm{TC}$ were merged for subsequent analysis. As shown in Table 1, patients with TT and TC/CC genotypes were well balanced with similar baseline characteristics.

\section{Associations Between rs2297/36 Polymorphism and Clinical Outcomes}

The best overall response of each patient was recorded for efficacy analysis. No complete response or partial response was observed in 26 cases, stable disease noted in 76, and progressive disease in 46 based on RECIST version 1.1. ORR was $17.6 \%$ (95\% CI 11.8\%-24.7\%) and diseasecontrol rate (DCR) 68.9\% (95\% CI 60.8\%-76.3\%). A waterfall plot for the best percentage change in target lesions is shown in Figure 2.

The last follow-up in this study was in September 2020. Median follow-up duration was 10.3 (1-36) months. A total of 142 patients had had PFS or had died at data cutoff. Therefore, PFS at data maturity was $95.9 \%$. As exhibited in Figure 3, median PFS patients

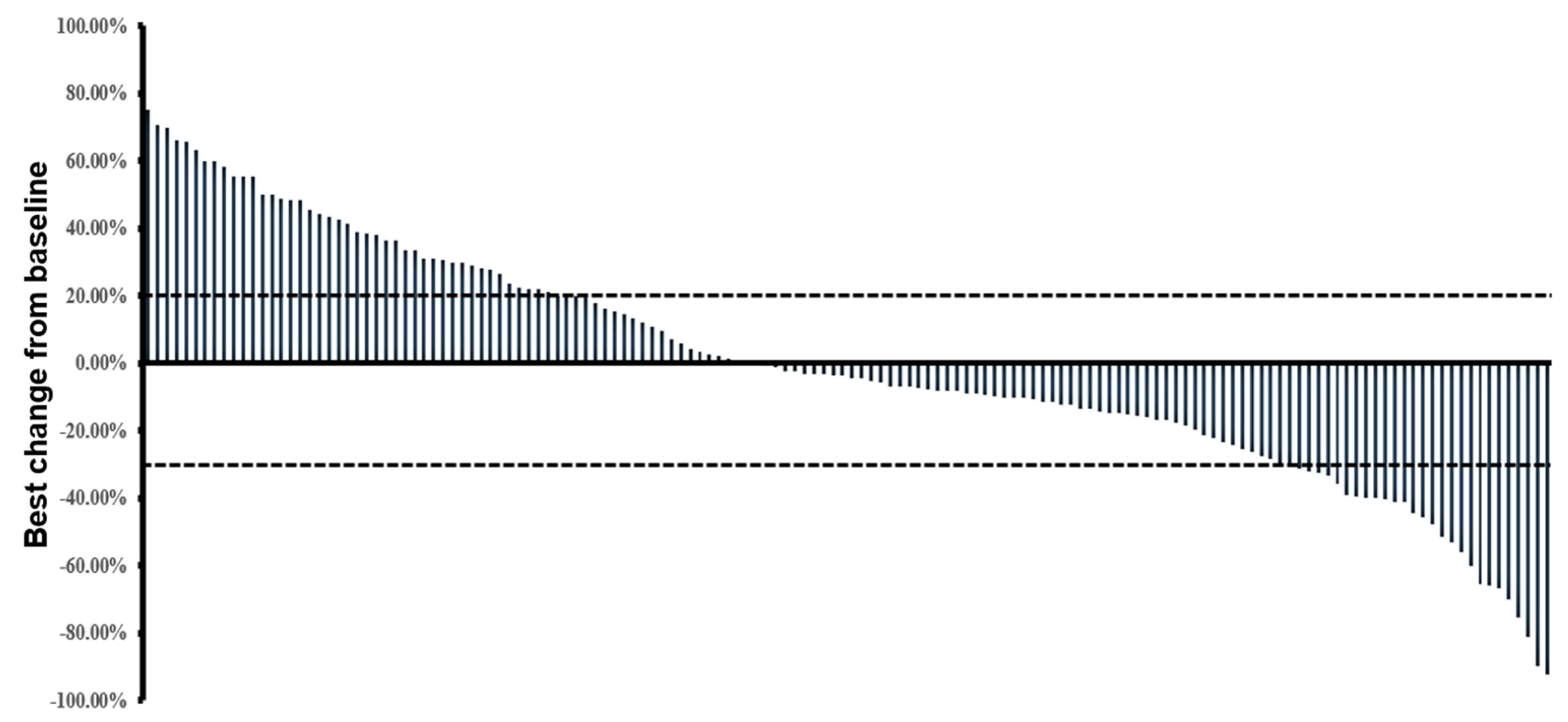

Figure 2 Waterfall plot of best change (reductions in sum of target-lesion diameters) from baseline.

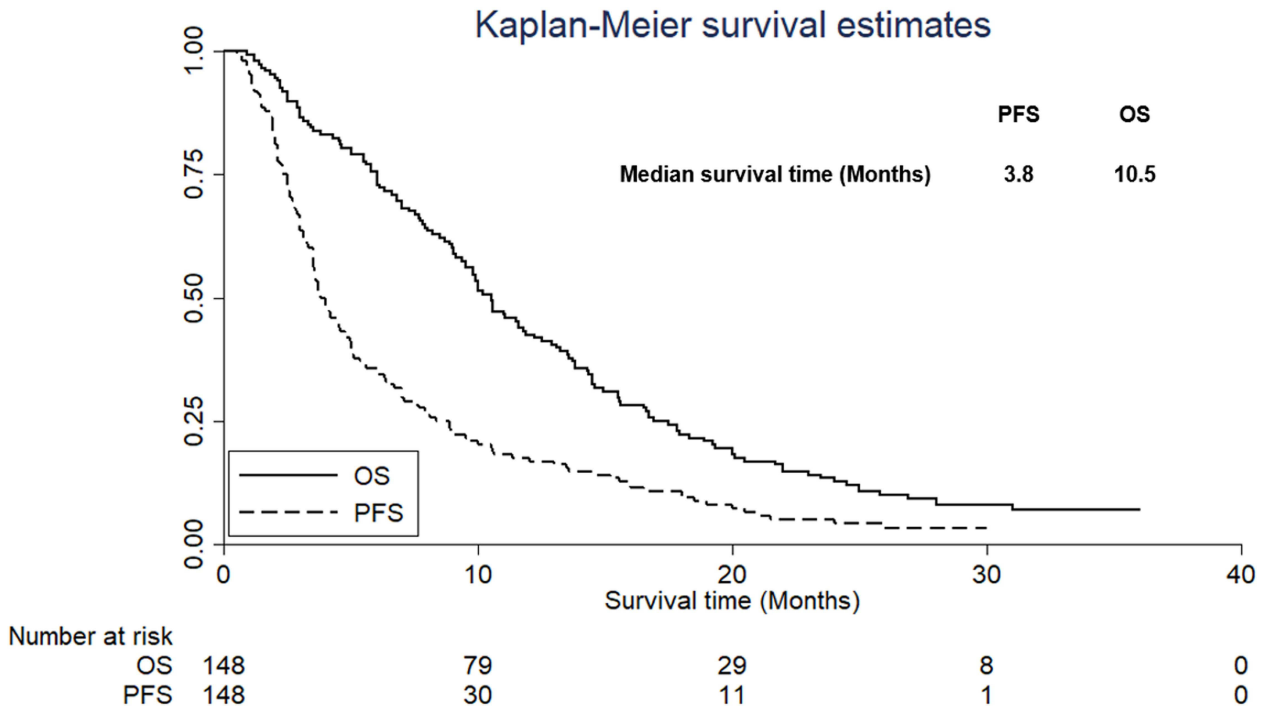

Figure 3 Progression-free survival and overall survival. 
Table 2 Univariate analysis of progression-free survival based on baseline characteristics

\begin{tabular}{|c|c|c|c|c|}
\hline & $\begin{array}{c}\text { Patients, } \\
\text { n }\end{array}$ & $\begin{array}{l}\text { Median } \\
\text { PFS }\end{array}$ & $95 \% \mathrm{Cl}$ & $P$ \\
\hline \multicolumn{5}{|l|}{ Age (years) } \\
\hline$<58$ & 71 & 4.0 & $3.35-4.65$ & 0.513 \\
\hline$\geq 58$ & 77 & 3.6 & $2.95-4.25$ & \\
\hline \multicolumn{5}{|l|}{ Sex } \\
\hline Male & 96 & 3.5 & $3.11-3.89$ & 0.311 \\
\hline Female & 52 & 4.1 & $3.05-5.15$ & \\
\hline \multicolumn{5}{|l|}{ Pathological stage } \\
\hline IIIB & 8 & 4.2 & $3.32-4.93$ & 0.635 \\
\hline IV & 140 & 3.7 & $3.10-4.22$ & \\
\hline \multicolumn{5}{|l|}{ ECOG score } \\
\hline 0 & 39 & 4.9 & $3.88-5.87$ & 0.018 \\
\hline $1-2$ & 109 & 3.2 & $2.85-3.55$ & \\
\hline \multicolumn{5}{|l|}{ Smoking status } \\
\hline $\begin{array}{l}\text { Nonsmoker/former } \\
\text { smoker }\end{array}$ & 107 & 4.1 & $3.25-4.95$ & 0.416 \\
\hline Smoker & 41 & 3.3 & $2.81-3.79$ & \\
\hline \multicolumn{5}{|l|}{ Histology } \\
\hline Adenocarcinoma & 114 & 3.9 & $3.15-4.65$ & 0.752 \\
\hline Squamous-cell carcinoma & 28 & 3.6 & $2.88-4.12$ & \\
\hline \multicolumn{5}{|l|}{$\begin{array}{l}\text { Number of } \\
\text { metastasis sites }\end{array}$} \\
\hline$\leq 3$ & 92 & 4.5 & $3.73-5.27$ & 0.025 \\
\hline$>3$ & 56 & 3.2 & $2.44-3.82$ & \\
\hline \multicolumn{5}{|l|}{$\begin{array}{l}\text { Driver-gene mutation } \\
\text { status }^{\text {a }}\end{array}$} \\
\hline Positive & 56 & 3.9 & $3.15-4.65$ & 0.554 \\
\hline Negative & 92 & 3.7 & $2.98-4.42$ & \\
\hline \multicolumn{5}{|l|}{$\begin{array}{l}\text { Previous systemic } \\
\text { treatment }\end{array}$} \\
\hline Second line & 43 & 4.2 & $3.34-5.06$ & 0.215 \\
\hline Further line & 105 & 3.6 & $3.11-4.09$ & \\
\hline \multicolumn{5}{|l|}{$\begin{array}{l}\text { History of targeted } \\
\text { drug therapy }\end{array}$} \\
\hline Yes & 92 & 3.8 & $3.01-4.59$ & 0.653 \\
\hline No & 56 & 3.7 & $2.98-4.42$ & \\
\hline \multicolumn{5}{|l|}{ History of antivascular } \\
\hline Yes & 10 & 3.5 & $2.86-4.14$ & 0.718 \\
\hline No & 138 & 3.9 & $3.24-4.56$ & \\
\hline \multicolumn{5}{|l|}{$\begin{array}{l}\text { Initial dosage of } \\
\text { apatinib (mg) }\end{array}$} \\
\hline 500 & 97 & 3.7 & $3.18-4.22$ & 0.616 \\
\hline 750 & 51 & 3.9 & $3.21-4.59$ & \\
\hline
\end{tabular}

Note: ${ }^{a}$ The driver gene were EGFR mutation or ALK or ROSI rearrangements Abbreviations: NSCLC, non-small cell lung cancer; ECOG, eastern cooperative oncology group; TKI, tyrosine kinase inhibitor; PFS, progression-free survival; $\mathrm{Cl}$, confidence interval. with NSCLC receiving apatinib was 3.8 (95\% CI 3.134.47) months. In terms of OS, 137 patients had died at data cutoff. Consequently, OS at data maturity was $92.6 \%$. As exhibited in Figure 3, median OS was 10.5 months $(95 \%$ CI 9.06-11.95).

We further analyzed PFS based on baselinecharacteristic subgroups, and univariate analysis of this is shown in Table 2. Obviously, ECOG score and number of metastasis sites were significantly associated with PFS: median PFS of patients with ECOG 0 was longer than those with ECOG $1-2$ score ( 4.9 vs 3.2 months, $P=0.018$ ). Median PFS of patients with number of metastasis sites fewer than or equal to was superior to those with greater than (4.5 vs 3.2, $P=0.025)$. As illustrated in Figure 4, median PFS of patients with TT and TC/CC genotypes of rs2297136 was 4.5 and 3.0 months, respectively $\left(\chi^{2}=7.69, P=0.006\right)$. Multivariate Cox regression model was used for PFS based on baseline characteristics significant on univariate analysis (Table 2) to adjust for confounding factors. Multivariate analysis results are shown in Table 3, with significant differences confirmed for the rs2297136 polymorphism and PFS, with rs2297136 an independent factor $(\mathrm{OR}=1.47, P=0.011)$. Interestingly, as shown in Table 3after adjustment in Cox regression analysis, ECOG score $(\mathrm{OR}=1.52, P=0.027)$ and number of metastasis sites $(\mathrm{OR}=1.39, P=0.039)$ were independent factors in PFS.

In terms of association between rs2297136-genotype status and OS, as exhibited in Figure 5, median OS of patients with TT and TC/CC genotypeswas 11.6 and 9.0 months, respectively $\left(\chi^{2}=3.98, P=0.04\right)$.

\section{Correlation Analysis Between PDLI rs2297/ 36 Polymorphism and Safety Profile of Patients}

The main adverse reactions during apatinib treatment were recorded and analyzed. As presented in Table 4, common adverse reactions related to apatinib treatment were hypertension, dermal toxicity, fatigue, oral toxicity, appetite decrease, diarrhea, nausea and vomiting, and hematologic toxicity, with incidence of $45.27 \%, 41.89 \%, 37.16 \%$, $31.76 \%, 31.08 \%, 24.32 \%, 17.57 \%$, and $11.49 \%$, respectively. As demonstrated in Table 4, no significant association was observed between adverse reactions and rs2297136-genotype status. 


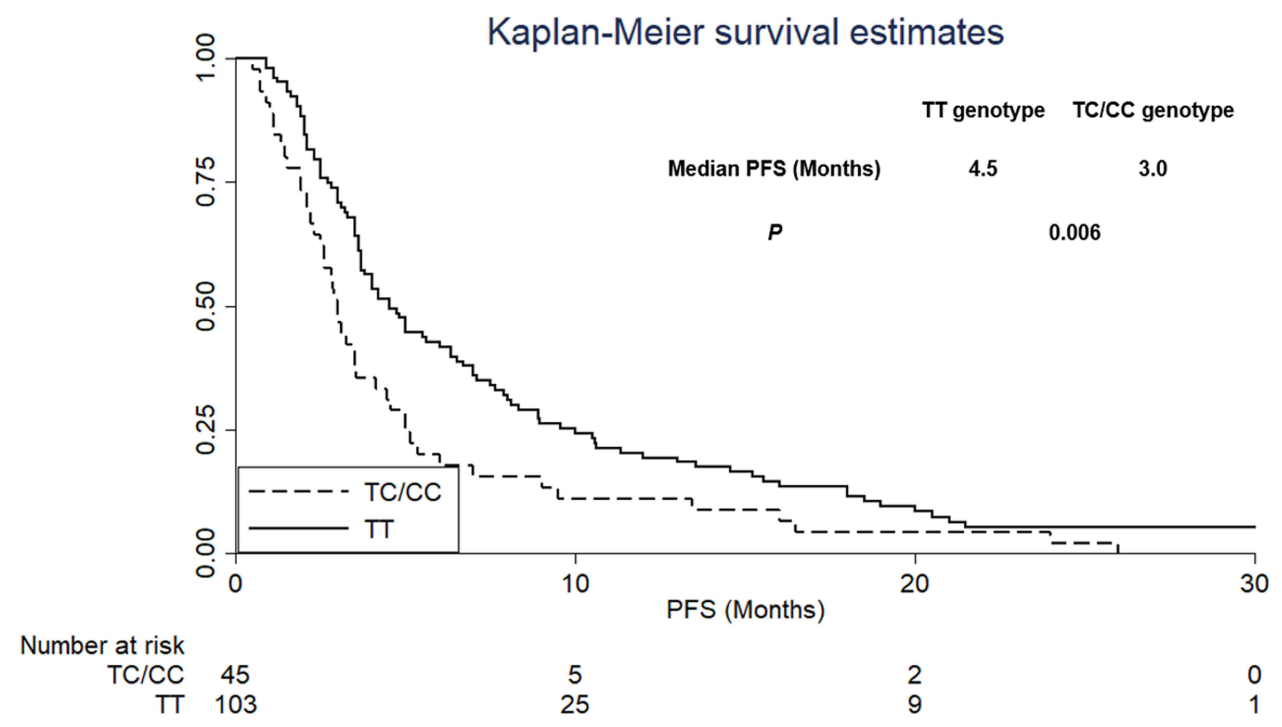

Figure 4 Progression-free survival based on PDLI rs2297/36 genotype status.

\section{Detection of PDL I-mRNA Expression and Prognosis Analysis According to PDLI -mRNA Status}

The genotype status of rs2297136 in the 68 specimens was TT genotype 47 cases $(69.12 \%)$, TC genotype 19 (27.94\%), CC genotype ywo (2.94\%), which was similar to the genotype-frequency distribution of the 148 patients. The distribution of the three genotypes corresponded with the Hardy-Weinberg equilibrium $(P=0.962)$ as well. CC and TC genotypes were merged in the subsequent analysis similarly. As exhibited in Figure 6, patients with the $\mathrm{TC} / \mathrm{CC}$ genotype showed higher relative expression of PDL1 mRNA in biopsied cancer-tissue specimens than those with the TT genotype (4.010 $\pm 0.517 \quad$ vs $2.703 \pm 0.677, \quad P<0.001)$. Additionally, PDL1-mRNA expression was divided into highand low PDL1 expressionbased onmedian PDL1- mRNA

Table 3 Multivariate Cox regression analysis for PFS based on baseline characteristics and rs2297/36 polymorphism status

\begin{tabular}{|l|l|l|c|}
\hline & OR $(95 \% \mathbf{~ C l})$ & df & $P$ \\
\hline $\begin{array}{l}\text { ECOG score } \\
\text { I-2 vs 0 }\end{array}$ & I.52 (I.09-2.02) & I & 0.027 \\
\hline $\begin{array}{l}\text { Number of metastasis sites } \\
>3 \text { vs } \leq 3\end{array}$ & $1.39(1.05-1.73)$ & $\mathrm{I}$ & 0.039 \\
\hline $\begin{array}{l}\text { PDLI rs2297I36 status } \\
\text { TC/CC vs TT }\end{array}$ & $\mathrm{I} .47(\mathrm{I} .1 \mathrm{I}-1.83)$ & $\mathrm{I}$ & $0.01 \mathrm{I}$ \\
\hline
\end{tabular}

expressionHigh and lowPDL1 were observed in 33 and 35 cases, respectively. As presented in Figure 7, patients with highPDL1 expression had a trend for worse PFS compared with those with low PDLlexpression (median PFS 3.5 vs 4.6), though difference was not statistically significant $\left(\chi^{2}=1.53, P=0.216\right)$.

\section{Discussion}

Our retrospective study provided real-world evidence regarding the efficacy and safety of apatinib monotherapy for patients with previously treated advanced NSCLC. Simultaneously, genetic variation analysis suggested that clinical outcomes of patients with advanced NSCLC receiving apatinib treatment could be influenced by the rs2297136 polymorphism through mediating the PDL1-mRNA expression.

The ORR and DCR of the 148 patients with NSCLC were $17.6 \%$ and $68.9 \%$, respectively. Median PFS was 3.8 months, slightly inferior to the first phase II clinical trial of apatinib in advanced NSCLC by Zhang et al in 2012 (ORR 20\%, DCR 68.89\%, and median PFS 4.7 months). ${ }^{23}$ This might be attributable to the retrospective design. The management of patients was inferior in retrospective study when compared with the clinical trials, which was demonstrated in the other retrospective study. ${ }^{24}$ These efficacy differences highlighted the gap between randomized controlled clinical trials and actual clinical practice. ${ }^{25}$ Patients with an ECOG score of 2 were included in our study as well. To our knowledge, the influence of ECOG score on prognosis has been 


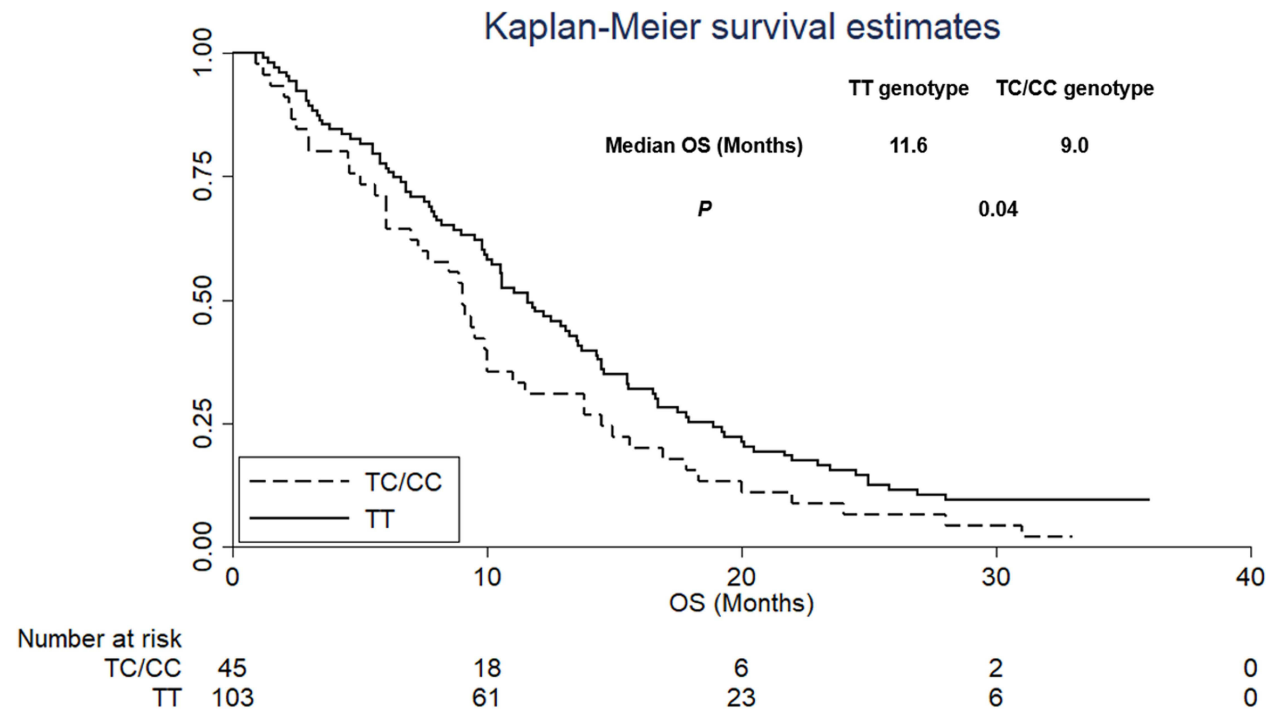

Figure 5 Overall survival based on PDLI rs2297I36 genotype status.

confirmed in many studies, which indicate that higher PS scores have worse prognoses. ${ }^{26}$ Results of the multivariate Cox analysis of this study suggested that patients with ECOG scores of 1-2 had worse prognoses compared with those with a score of 0 . However, it should be noted that median OS of our study was 10.5 months, which was slightly longer than the median OS of another apatinib study reported recently. ${ }^{22}$ The reason for this could be the continued license for PD1/PDL1 inhibitors and anlotinib in China since 2018. ${ }^{10}$ Patients with advanced NSCLC stood a good chance to choose PD1/PDL1 inhibitors and targeted drugs as subsequent treatment, which have proven to be effective and brought significant survival benefits. $^{27}$
This study is the first study to focus on the correlation between the efficacy of apatinib treatment in patients with advanced NSCLCand PDL1-polymorphism analysis in Chinese patients. Interestingly, a recent study initiated by Wang et al investigated clinical outcomes and safety of apatinib in the treatment of advanced nonsquamous NSCLC and the clinical significance of KDR-gene polymorphism. ${ }^{22}$ The results suggested that clinical outcomes of treatment with apatinib might be influenced by the KDR 4397T $>$ C polymorphism. The conclusion of that study highlighted the potential predictive value of pharmacogenomics in the clinical outcomes of patients with NSCLC receiving apatinib treatment. In terms of the polymorphism analysis in our study, minor-allele frequency of

Table 4 Correlation analysis between PDLI rs2297I36 polymorphism and safety profile of apatinib administration

\begin{tabular}{|c|c|c|c|c|c|c|}
\hline \multirow[t]{2}{*}{ Adverse events } & \multirow[t]{2}{*}{ Grade I-2 } & \multirow[t]{2}{*}{ Grade 3-4 } & \multirow[t]{2}{*}{ Total, (\%) } & \multicolumn{2}{|c|}{ rs2297 I 36 genotype status } & \multirow[t]{2}{*}{$\boldsymbol{P}$} \\
\hline & & & & TT $(n=103)$ & $\operatorname{TC} / C C(n=45)$ & \\
\hline Hypertension & $50(33.78)$ & I7 (I I.49) & $67(45.27)$ & $46(44.66)$ & $21(46.67)$ & 0.822 \\
\hline Dermal toxicity & $5 \mathrm{I}(34.46)$ & II (7.43) & $62(41.89)$ & $44(42.72)$ & $18(40.00)$ & 0.758 \\
\hline Fatigue & $51(34.46)$ & $4(2.70)$ & $55(37.16)$ & $40(38.83)$ & $15(33.33)$ & 0.524 \\
\hline Oral toxicity & $45(30.4 I)$ & $2(1.35)$ & $47(31.76)$ & $33(32.04)$ & $14(3 \mathrm{I} .1 \mathrm{I})$ & 0.911 \\
\hline Appetite decreased & $43(29.05)$ & $3(2.03)$ & $46(31.08)$ & $34(33.01)$ & $12(26.67)$ & 0.443 \\
\hline Diarrhea & $32(21.62)$ & $4(2.70)$ & $36(24.32)$ & $27(26.21)$ & $9(20.00)$ & 0.418 \\
\hline Nausea and vomiting & $26(17.57)$ & 0 & $26(17.57)$ & $19(18.36)$ & $7(15.56)$ & 0.671 \\
\hline Hematologic toxicity & $15(10.14)$ & $2(1.35)$ & $17(11.49)$ & $10(9.71)$ & $7(15.56)$ & 0.305 \\
\hline
\end{tabular}




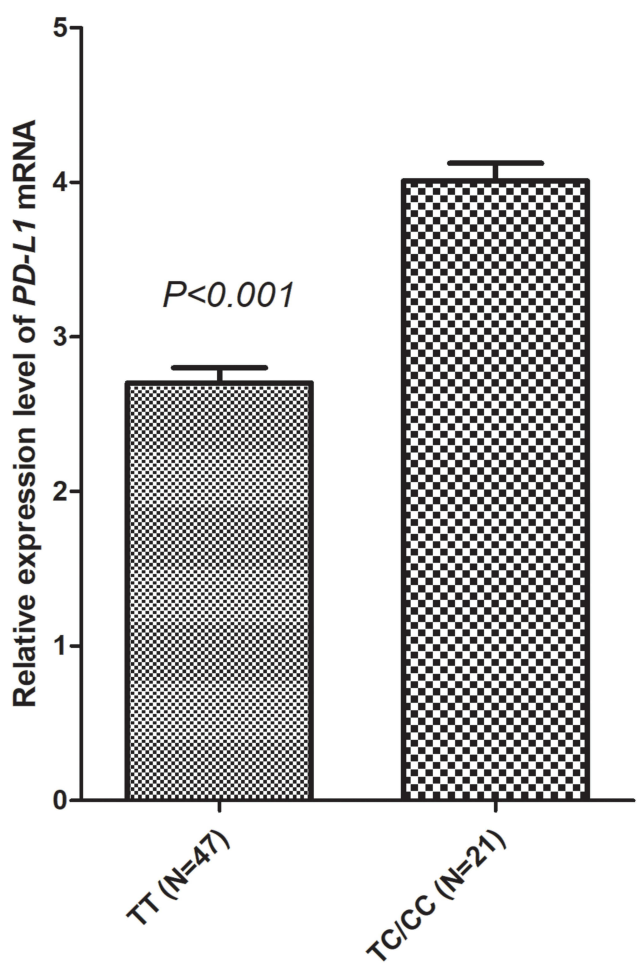

Figure 6 Relative expression level of PDLI mRNA based on PDLI rs2297136 genotype status.

rs2297136 was 0.17 , in line with the genotype frequency of the Chinese population in the NCBI database and the results of $\mathrm{Xie}$ et $\mathrm{al}^{28}$ Minor-allele frequency in the Caucasian population is significantly different from that in our study, which suggests that great ethnic differences in PDL1 polymorphisms exist clinically. ${ }^{29}$
Xie et al studied 225 hepatocellular carcinoma patients, and their results suggested that the CC genotype of rs2297136 was correlated with predisposition to this cancer type. Their prognosis-analysis results were basically consistent with the results of our study. They also showed that patients with minor alleles of rs2297136 had worse prognoses. Many studies have suggested that PDL1 polymorphisms are associated with the risk of cancer. ${ }^{30}$

Recently, a retrospective analysis by $\mathrm{Wu}$ et al explored the association between genetic variation of $P D L 1$ and its protein expression and prognosis in gastric cancer. $^{17}$ A total of 728 gastric cancer patients were included in their polymorphism analysis, and the results suggested that rs2297136genotype A was correlated with PDL1 protein expression and superior prognosis in patients without postoperative chemotherapy, consistent with the results of our study to some extent, considering that only $P D L 1$-mRNA expression was assessed in ours. Previous research has confirmed that PDL1mRNA expression is positively correlated with PDL1 protein expression among patients with EGFR-mutated lung adenocarcinoma. ${ }^{31} \mathrm{Du}$ et al explored the association between rs2297136 and predisposition toNSCLC. ${ }^{32}$ The results showed that rs2297136 influenced predisposition to NSCLC. A recent real-world studywas conducted on 315 patients with R0-resected colorectal cancer who received capecitabine-based adjuvant chemotherapy. ${ }^{18}$ The results indicated that the $901 \mathrm{~T}>\mathrm{C}$ TC/CC genotype conferred significantly higher mRNA

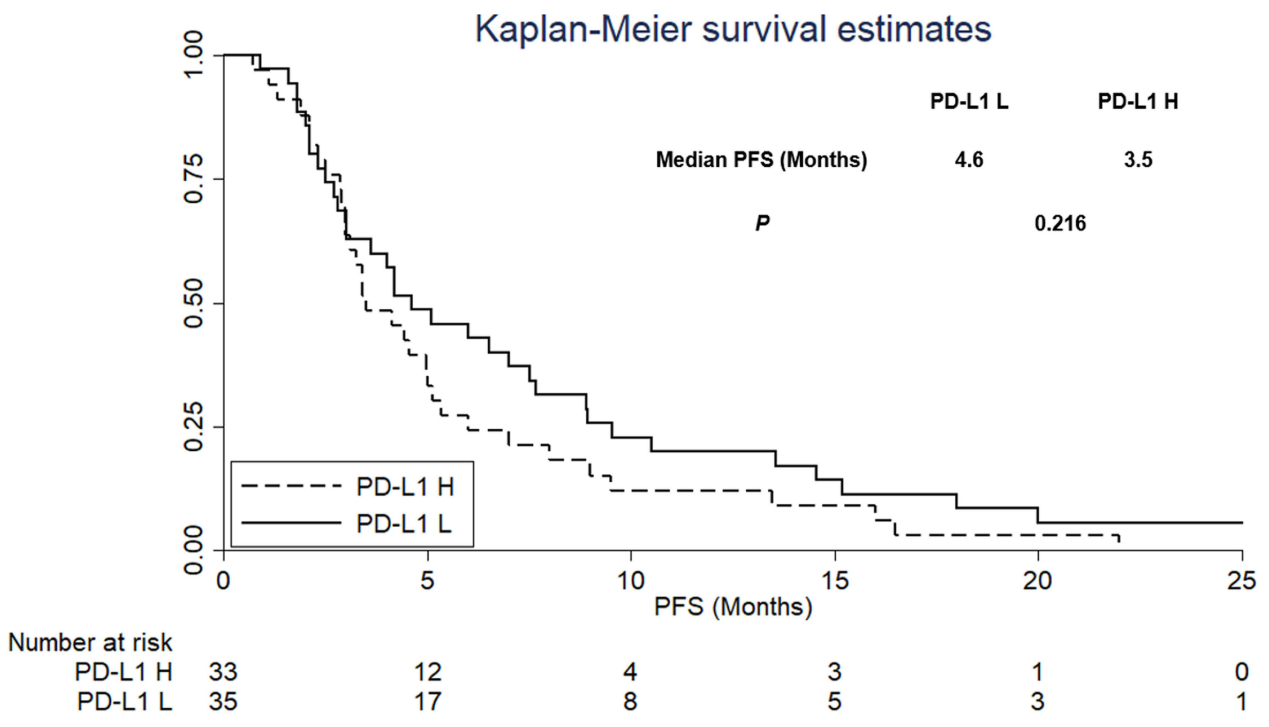

Figure 7 Progression-free survival of the 68 specimens from patients based on PDLI mRNA-expression status. 
expression than those with the TT genotype, which is consistent with the mRNA-expression results in our study. Additionally, it should be noted that rs2297136 was located at PDL1 3'UTR, which can cause disruption in miRNA attaching to this region, perhaps leading to altered $P D L 1$ expression at the protein level and be of prognostic significance for patients with NSCLC receiving apatinib treatment. In terms of safety profile, the common adverse reactions were hypertension, dermal toxicity, fatigue, oral toxicity, appetite decrease, diarrhea, nausea and vomiting, and hematologic toxicity, similar to the results of previous retrospective studies of apatinib regimens. ${ }^{22,33}$ The correlation analysis between the polymorphism and adverse reactions failed to show a significant difference.

Noteworthily, PDL1 is a hot-spot gene for efficacy predication of $P D 1 / P D L 1$ inhibitors. ${ }^{34}$ However, the clinical significance of $P D L 1$ expression in the prognosis of patients with advanced NSCLC receiving antiangiogenic drugs instead of immunotherapy is still unknown and thus necessary to investigate. ${ }^{35}$ The results of our study preliminarily suggested that patients with high mRNA expression of $P D L 1$ might have worse prognosis, in line with previous studies in which patients with high $P D L 1$ expression demonstrated worse prognosis when received EGFR TKIs $^{36}$ and concurrent chemoradiotherapy treatment. ${ }^{37}$ However, the gene's role in vivo is still unclear. Several clinical studies indicated that patients with high expression of PDL1 had better prognosis. ${ }^{38}$ These results might be attributed to the heterogeneity of patients included. Briefly, further in-depth prospective clinical trials are needed to explore the clinical significance of $P D L 1$ polymorphisms.

We believe our study is of potential clinical significance for prognosis evaluation of patients with advanced NSCLC who have been treated with apatinib. Further randomized controlled clinical trials are warranted to validate the conclusions.

\section{Conclusion}

The present retrospective study provided real-world evidence regarding the superior efficacy and tolerable adverse reactions of apatinib monotherapy for patients with previously treated advanced NSCLC. Also, PDL1 polymorphism rs2297136 could be used as a potential biomarker for prognosis of patients with NSCLC receiving apatinib treatment.

\section{Acknowledgments}

The authors would like to express their sincere gratitude to the patients and their families for participating in this study. We thank all the staff who took part in this study.

\section{Disclosure}

The authors declare that there are no conflicts of interest.

\section{References}

1. Xu K, Zhang C, Du T, et al. Progress of exosomes in the diagnosis and treatment of lung cancer. Biomed Pharmacother. 2021;134:111111. doi:10.1016/j.biopha.2020.111111.

2. Zhang J, Mao W, Chen Z, Gu H, Lian C. Clinical Significance of Has_circ_0060937 in Bone Metastasis of NSCLC. Int J Gen Med. 2020;13:1115-1121. doi:10.2147/ijgm.s279023.

3. Phan TT, Ho TT, Nguyen HT, et al. The prognostic impact of neutrophil to lymphocyte ratio in advanced non-small cell lung cancer patients treated with EGFR TKI. Int $J$ Gen Med. 2018;11:423-430. doi:10.2147/ijgm.s174605.

4. Arbour KC, Riely GJ. Systemic Therapy for Locally Advanced and Metastatic Non-Small Cell Lung Cancer: a Review. JAMA. 2019;322 (8):764-774. doi:10.1001/jama.2019.11058.

5. Ghadyalpatil NS, Pandey A, Krishnamani I, et al. First-line management of metastatic non-small cell lung cancer: an Indian perspective. South Asian J Cancer. 2019;8(2):73-79. doi:10.4103/sajc.sajc_294_18.

6. Garassino MC, Kawaguchi T, Gregorc V, et al. Chemotherapy versus erlotinib as second-line treatment in patients with advanced non-small cell lung cancer and wild-type epidermal growth factor receptor: an individual patient data (IPD) analysis. ESMO Open. 2018;3(6):e000327. doi:10.1136/esmoopen-2018-000327.

7. Huang L, Li L, Zhou Y, et al. Clinical Characteristics Correlate With Outcomes of Immunotherapy in Advanced Non-Small Cell Lung Cancer. J Cancer. 2020;11(24):7137-7145. doi:10.7150/jca.49213.

8. Folkman J. Tumor angiogenesis: therapeutic implications. $N$ Engl J Med. 1971;285(21):1182-1186. doi:10.1056/nejm197111182852108.

9. Manzo A, Montanino A, Carillio G, et al. Angiogenesis Inhibitors in NSCLC. Int J Mol Sci. 2017;18(10):10. doi:10.3390/ijms18102021.

10. Han B, Li K, Wang Q, et al. Effect of Anlotinib as a Third-Line or Further Treatment on Overall Survival of Patients With Advanced Non-Small Cell Lung Cancer: the ALTER 0303 Phase 3 Randomized Clinical Trial. JAMA Oncol. 2018;4(11):1569-1575. doi:10.1001/ jamaoncol.2018.3039.

11. Li J, Qin S, Xu J, et al. Randomized, Double-Blind, Placebo-Controlled Phase III Trial of Apatinib in Patients With Chemotherapy-Refractory Advanced or Metastatic Adenocarcinoma of the Stomach or Gastroesophageal Junction. J Clin Oncol. 2016;34(13):1448-1454. doi:10.1200/jco.2015.63.5995.

12. Zhao D, Hou H, Zhang X. Progress in the treatment of solid tumors with apatinib: a systematic review. Onco Targets Ther. 2018;11:4137-4147. doi:10.2147/ott.s172305.

13. Villaruz LC, Socinski MA. The role of anti-angiogenesis in non-small-cell lung cancer: an update. Curr Oncol Rep. 2015;17 (6):26. doi:10.1007/s11912-015-0448-y.

14. Hudson K, Cross N, Jordan-Mahy N, Leyland R. The Extrinsic and Intrinsic Roles of PD-L1 and Its Receptor PD-1: implications for Immunotherapy Treatment. Front Immunol. 2020;11:568931. doi:10.3389/fimmu.2020.568931.

15. Paz-Ares L, Luft A, Vicente D, et al. Pembrolizumab plus Chemotherapy for Squamous Non-Small-Cell Lung Cancer. $N$ Engl J Med. 2018;379(21):2040-2051. doi:10.1056/NEJMoa1810865. 
16. Yi M, Jiao D, Qin S, et al. Synergistic effect of immune checkpoint blockade and anti-angiogenesis in cancer treatment. Mol Cancer. 2019;18(1):60. doi:10.1186/s12943-019-0974-6.

17. Wu Y, Zhao T, Jia Z, et al. Polymorphism of the programmed death-ligand 1 gene is associated with its protein expression and prognosis in gastric cancer. J Gastroenterol Hepatol. 2019;34 (7):1201-1207. doi:10.1111/jgh.14520.

18. Su J, Dai B, Yuan W, et al. The influence of PD-L1 genetic variation on the prognosis of R0 resection colorectal cancer patients received capecitabine-based adjuvant chemotherapy: a long-term follow-up, real-world retrospective study. Cancer Chemother Pharmacol. 2020;85(5):969-978. doi:10.1007/s00280-020-04069-1.

19. Flaifel A, Xie W, Braun DA, et al. PD-L1 Expression and Clinical Outcomes to Cabozantinib, Everolimus, and Sunitinib in Patients with Metastatic Renal Cell Carcinoma: analysis of the Randomized Clinical Trials METEOR and CABOSUN. Clin Cancer Res. 2019;25 (20):6080-6088. doi:10.1158/1078-0432.ccr-19-1135.

20. Eisenhauer EA, Therasse P, Bogaerts J, et al. New response evaluation criteria in solid tumours: revised RECIST guideline (version 1.1). Eur $J$ Cancer. 2009;45(2):228-247. doi:10.1016/j. ejca.2008.10.026.

21. Miller TP, Fisher BT, Getz KD, et al. Unintended consequences of evolution of the Common Terminology Criteria for Adverse Events. Pediatr Blood Cancer. 2019;66(7):e27747. doi:10.1002/pbc.27747.

22. Song ZZ, Zhao LF, Zuo J, et al. Clinical Outcomes and Safety of Apatinib Mesylate in the Treatment of Advanced Non-Squamous Non-Small Cell Lung Cancer in Patients Who Progressed After Standard Therapy and Analysis of the KDR Gene Polymorphism. Onco Targets Ther. 2020;13:603-613. doi:10.2147/ott.s222985.

23. Ma JT, Sun J, Sun L, et al. Efficacy and safety of apatinib in patients with advanced nonsmall cell lung cancer that failed prior chemotherapy or EGFR-TKIs: a pooled analysis. Medicine. 2018;97(35): e12083. doi: $10.1097 / \mathrm{md} .0000000000012083$.

24. Fang SC, Huang W, Zhang YM, Zhang HT, Xie WP. Hypertension as a predictive biomarker in patients with advanced non-small-cell lung cancer treated with apatinib. Onco Targets Ther. 2019;12:985-992. doi: $10.2147 /$ ott.s189984.

25. Zhang D, Zhang C, Huang J, Guan Y, Guo Q. Clinical investigation of the efficacy and toxicity of apatinib (YN968D1) in stage III/IV non-small cell lung cancer after second-line chemotherapy treatment: a retrospective study. Thorac Cancer. 2018;9(12):1754-1762. doi:10.1111/1759-7714.12898.

26. Agemi Y, Shimokawa T, Sasaki J, et al. Prospective evaluation of the G8 screening tool for prognostication of survival in elderly patients with lung cancer: a single-institution study. PLoS One. 2019;14(1): e0210499. doi:10.1371/journal.pone.0210499.

27. Nadal E, Massuti B, Domine M, et al. Immunotherapy with checkpoint inhibitors in non-small cell lung cancer: insights from long-term survivors. Cancer Immunol Immunother. 2019;68 (3):341-352. doi:10.1007/s00262-019-02310-2.
28. Xie Q, Chen Z, Xia L, et al. Correlations of PD-L1 gene polymorphisms with susceptibility and prognosis in hepatocellular carcinoma in a Chinese Han population. Gene. 2018;674:188-194. doi:10.1016/j. gene.2018.06.069.

29. Catalano C, da Silva Filho MI, Frank C, et al. Investigation of single and synergic effects of NLRC5 and PD-L1 variants on the risk of colorectal cancer. PLoS One. 2018;13(2):e0192385. doi:10.1371/ journal.pone.0192385.

30. Hashemi M, Karami S, Sarabandi S, et al. Association between PD-1 and PD-L1 Polymorphisms and the Risk of Cancer: a Meta-Analysis of Case-Control Studies. Cancers. 2019;11(8):1150. doi:10.3390/ cancers 11081150.

31. Isobe K, Kakimoto A, Mikami T, et al. PD-L1 mRNA expression in EGFR-mutant lung adenocarcinoma. Oncol Rep. 2018;40 (1):331-338. doi:10.3892/or.2018.6442.

32. Du W, Zhu J, Chen Y, et al. Variant SNPs at the microRNA complementary site in the B7H1 3'untranslated region increase the risk of nonsmall cell lung cancer. Mol Med Rep. 2017;16(3):2682-2690. doi:10.3892/mmr.2017.6902.

33. Zhang Z, Zhao Y, Lu F, et al. Multi-targeted tyrosine kinase inhibitors as third-line regimen in advanced non-small cell lung cancer: a network meta-analysis. Ann Transl Med. 2019;7(18):452. doi:10.21037/atm.2019.08.51.

34. Miyawaki E, Murakami H, Mori K, et al. PD-L1 expression and response to pembrolizumab in patients with EGFR-mutant non-small cell lung cancer. Jpn J Clin Oncol. 2020;50(5):617-622. doi:10.1093/jjco/hyaa033.

35. Xu Y, Wan B, Chen X, et al. The association of PD-L1 expression with the efficacy of anti-PD-1/PD-L1 immunotherapy and survival of non-small cell lung cancer patients: a meta-analysis of randomized controlled trials. Transl Lung Cancer Res. 2019;8(4):413-428. doi:10.21037/tlcr.2019.08.09.

36. Kim T, Cha YJ, Chang YS. Correlation of PD-L1 Expression Tested by $22 \mathrm{C} 3$ and SP263 in Non-Small Cell Lung Cancer and Its Prognostic Effect on EGFR Mutation-Positive Lung Adenocarcinoma. Tuberc Respir Dis. 2020;83(1):51-60. doi: $10.4046 / \operatorname{trd} .2019 .0026$.

37. Gennen K, Kasmann L, Taugner J, et al. Prognostic value of PD-L1 expression on tumor cells combined with CD8+ TIL density in patients with locally advanced non-small cell lung cancer treated with concurrent chemoradiotherapy. Radiat Oncol. 2020;15(1):5. doi:10.1186/s13014-019-1453-3.

38. Berntsson J, Eberhard J, Nodin B, et al. Expression of programmed cell death protein 1 (PD-1) and its ligand PD-L1 in colorectal cancer: relationship with sidedness and prognosis. Oncoimmunology. 2018;7 (8):e1465165. doi:10.1080/2162402x.2018.1465165.
International Journal of General Medicine

\section{Publish your work in this journal}

The International Journal of General Medicine is an international, peer-reviewed open-access journal that focuses on general and internal medicine, pathogenesis, epidemiology, diagnosis, monitoring and treatment protocols. The journal is characterized by the rapid reporting of reviews, original research and clinical studies across all disease areas. The manuscript management system is completely online and includes a very quick and fair peer-review system, which is all easy to use. Visit http://www.dovepress.com/ testimonials.php to read real quotes from published authors. 